\title{
TSLP protects against liver I/R injury via activation of the PI3K/Akt pathway
}

\author{
Shilai Li, ${ }^{1,2}$ Zhongjie Yi, ${ }^{1,3}$ Meihong Deng, ${ }^{1}$ Melanie J. Scott, ${ }^{1}$ Chenxuan Yang, ${ }^{1,4}$ Wenbo Li, ${ }^{1,5}$ \\ Zhao Lei, ${ }^{1,3}$ Nicole M. Santerre, ${ }^{1}$ Patricia Loughran, ${ }^{1,6}$ and Timothy R. Billiar ${ }^{1}$ \\ 'Department of Surgery, University of Pittsburgh, Pittsburgh, Pennsylvania, USA. ${ }^{2}$ Department of Emergency, The First \\ Affiliated Hospital of Guangxi Medical University, Nanning, China. ${ }^{3}$ Department of Hepatobiliary Surgery, The Third \\ Xiangya Hospital, Central South University, Changsha, China. ${ }^{4}$ School of Medicine, Tsinghua University, Beijing, China. \\ ${ }^{5}$ Department of Plastic Surgery, The Second Xiangya Hospital, Central South University, Changsha, China. ${ }^{6}$ Center for \\ Biologic Imaging, University of Pittsburgh, Pennsylvania, USA.
}

Thymic stromal lymphopoietin (TSLP) is a cytokine mainly released by epithelial cells that plays important roles in inflammation, autoimmune disease, and cancer. While TSLP is expressed in the liver at high levels, the role of TSLP in liver ischemia/reperfusion (I/R) injury remains unknown. Experiments were carried out to determine the role of TSLP in liver I/R injury. Wild-type (WT) and TSLP receptor-knockout (TSLPR ${ }^{-1-}$ ) mice were subjected to liver partial warm I/R injury. Liver injury was assessed by measuring serum alanine aminotransferase (ALT) level, necrotic areas by liver histology, hepatocyte death, and local hepatic inflammatory responses. Signal pathways were explored in vivo and in vitro to identify possible mechanisms for TSLP in I/R injury. TSLP and TSLPR protein expression increased during liver I/R in vivo and following hepatocyte hypoxia/ reoxygenation in vitro. Deletion of TSLPR or neutralization of TSLP with anti-TSLP antibody exacerbated liver injury in terms of serum ALT levels as well as necrotic areas in liver histology. Administration of exogenous recombinant mouse TSLP to WT mice significantly reduced liver damage compared with controls, but failed to prevent I/R injury in TSLPR ${ }^{-/-}$mice. TSLP induced autophagy in hepatocytes during liver I/R injury. Mechanistically, Akt was activated in WT mice during liver I/R injury. The opposite results were observed in TSLPR ${ }^{-/-}$mice. In addition, TSLP could directly induce Akt activation in hepatocytes independent of nonparenchymal cells in vitro. Furthermore, the Akt agonist, insulin-like growth factor-1 (IGF-1), prevented I/R injury in TSLPR ${ }^{-1-}$ mice and an Akt inhibitor, LY294002, blocked the protective effects of TSLP in WT mice subjected to I/R. Our data indicate that TSLP protects against liver I/R injury via activation of the PI3K/Akt pathway. Through this pathway, TSLP induces autophagy in hepatocytes. Thus, TSLP is a potent inhibitor of stress-induced hepatocyte necrosis.

Authorship note: SL and ZY contributed equally to this work.

Conflict of interest: The authors have declared that no conflict of interest exists.

Copyright: (c) 2019, American Society for Clinical Investigation.

Submitted: March 25, 2019

Accepted: October 10, 2019

Published: November 14, 2019

Reference information: JCI Insight. 2019;4(22):e129013.

https://doi.org/10.1172/jci.

insight.129013.

\section{Introduction}

Liver ischemia/reperfusion ( $\mathrm{I} / \mathrm{R}$ ) injury occurs in many clinical scenarios, including liver transplantation, trauma, hemorrhagic shock, and liver resections (1). While techniques for safer liver surgery have improved surgical outcomes, liver I/R injury is still the major cause of postoperative liver dysfunction and failure, especially in liver transplantation (2). Despite efforts to understand the mechanisms responsible for liver I/R injury, we have only a partial understanding of the molecular and cellular events that regulate liver injury following $\mathrm{I} / \mathrm{R}$. Oxidative stress is thought to initiate injury during the ischemic phase and also initiate a sterile inflammatory response that manifests following reperfusion, which contributes to further liver injury. The combined effects of oxidative stress and excessive inflammation lead to hepatocyte necrosis and apoptosis (3).

Thymic stromal lymphopoietin (TSLP) is a member of the IL-2 cytokine family, and a distant paralog of IL-7 (4). It is mainly released by epithelial cells, and it can also be produced by fibroblasts, smooth muscle cells, and immune cells under mechanical injury and in inflammatory conditions (5). TSLP is known to be highly expressed in heart, liver, and prostate. The low-affinity TSLP receptor (TSLPR) and IL-7 receptor $\alpha$ chain combine to form a high-affinity TSLP heterodimeric receptor complex (6). TSLPR mainly distributes on immune cells including lymphocytes, dendritic cells (DCs), and monocytes. Previous studies 
have suggested that TSLP is involved in immune disorders including allergic inflammation (7), infection (8), cancer (9), and autoimmunity (10). Recent studies suggest that TSLP is involved in innate and adaptive immune responses $(5,11)$. While it is known that liver cells including hepatocytes can produce TSLP, it remains unknown if TSLP is involved in liver I/R injury.

In this study, we evaluated the hypothesis that TSLP is involved in the pathogenesis of liver I/R injury. Wild-type (WT) mice and TSLP receptor-knockout $\left(\mathrm{TSLPR}^{-/}\right)$mice were subjected to liver I/R injury. Both TSLP and TSLPR were rapidly upregulated by ischemia in the liver and hypoxia in hepatocytes, and $\mathrm{TSLPR}^{-/}$mice exhibited much worse liver damage after I/R compared with WT mice. Furthermore, TLSP protected against liver I/R injury and hypoxic cell death via activation of the PI3K/Akt pathway and the activation of autophagy in hepatocytes. Thus, exogenously administered TSLP might be useful to prevent injury after liver I/R.

\section{Results}

$T S L P$ and TSLPR protein expression increased after liver $I / R$ injury in vivo and hypoxia/reoxygenation in vitro. To determine whether liver TSLP or TSLPR levels change after liver I/R, we subjected WT mice to partial liver ischemia followed by reperfusion. TSLP and TSLPR proteins were expressed at low levels at baseline but were induced substantially during liver I/R. Liver TSLP levels were markedly elevated above baseline by 1 hour of ischemia and before reperfusion. TSLP levels remained elevated throughout the 24-hour observation time period. Increases in TSLPR levels were observed within 1 hour; however, reperfusion induced further increases that remained through the 24 hours of observation (Figure 1A). Interestingly, deletion of TSLPR resulted in higher levels of TSLP in the liver at baseline, as measured by Western blot or ELISA. While TSLP levels increased in the livers of WT mice, levels remained even higher in the livers of $\mathrm{TSLPR}^{-/-}$mice at 6 hours after the initiation of I/R (Figure 1B).

To further evaluate the origins of the elevated TSLP and TSLPR expression, we mimicked I/R in vitro by subjecting cultured hepatocytes and nonparenchymal cells to hypoxia for 10 hours ( $1 \%$ oxygen) followed by reoxygenation every 2 hours for an additional 12 hours $(0,2,4,6,8,10$, and 12 hours). TSLP and TSLPR protein expression increased substantially in hepatocytes and nonparenchymal cells, as assessed by Western blot; however, the relative increase was much greater in hepatocytes (Figure 1, C and D). TSLP levels also increased in the supernatants of cultured hepatocytes at 12 hours after H/R (Figure 1E). The elevations of TSLP and TSLPR expression in vivo and in vitro in liver cells with ischemia suggest the possible involvement of TSLP during liver I/R injury.

TSLP signaling protects against liver $I / R$ injury. To determine the role of TSLP in liver I/R injury we subjected WT and $\mathrm{TSLPR}^{-/-}$mice to liver I/R injury and assessed liver injury by measuring serum alanine aminotransferase (ALT) levels at 0, 1, 3, 6, and 24 hours after 1 hour of ischemia. As shown in Figure 2A, $\mathrm{TSLPR}^{-/-}$mice exhibited higher ALT levels starting at 1 hour after reperfusion, which persisted to 6 hours. By 24 hours ALT levels had dropped to similar levels in both WT and TSLPR ${ }^{-1}$ mice. Morphological indexes (hematoxylin and eosin [H\&E] staining) were assessed at 6 hours after reperfusion and confirmed that the necrotic areas of the ischemic hepatic lobes were significantly greater in TSLPR ${ }^{-/}$mice when compared with WT mice (Figure 2B). These results indicate that TSLPR deficiency exacerbates liver I/R injury.

To confirm the protective effects of TSLP in liver I/R injury, we next neutralized TSLP with anti-TSLP antibody in WT mice during I/R. WT mice subjected to liver I/R injury were treated with anti-TSLP antibody or IgG immediately after reperfusion $(100 \mu \mathrm{g} /$ mouse, intraperitoneally [i.p.]). Liver damage was markedly exacerbated in the anti-TSLP antibody group when compared with the IgG group, as assessed by serum ALT levels and necrotic areas measured by histology (Figure 2, C and D). Considering that TSLPR deficiency and neutralizing TSLP exacerbated liver I/R injury, we concluded that TSLP protects against liver I/R injury.

To determine if exogenous TSLP could protect from I/R injury, we subjected WT and TSLPR ${ }^{-/}$mice to liver I/R and treated the mice with mouse recombinant TSLP (rTSLP) or phosphate-buffered saline (PBS) immediately after reperfusion ( $2 \mu \mathrm{g}$ /mouse, i.p.). WT mice treated with rTSLP showed dramatically attenuated serum ALT levels and liver necrosis after liver I/R when compared with the mice treated with PBS (Figure 2, E and F). In contrast, TSLPR ${ }^{-/}$mice treated with rTSLP showed serum ALT levels and necrotic areas after liver I/R similar to those of mice treated with PBS (Figure 2, G and H). Taken together, these data demonstrate that TSLP protects against liver I/R injury via its receptor TSLPR.

TSLP induces autophagy in vivo and in vitro. Cell death is the end result of liver I/R injury, and can propagate injury through the activation of inflammatory pathways. Activation of autophagy can prevent cell 


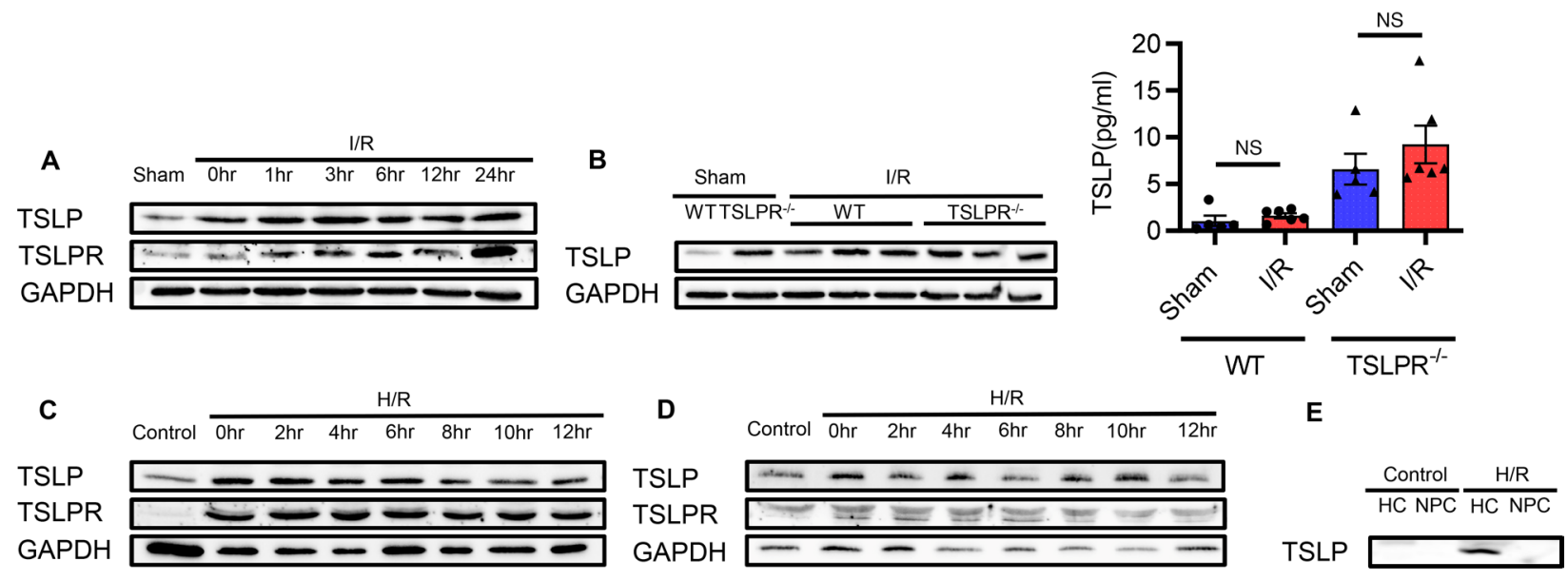

Figure 1. TSLP and TSLPR protein expression increase after liver I/R injury in vivo and $\mathbf{H} / \mathbf{R}$ in vitro. (A) TSLP and TSLPR protein expression in liver from WT mice in sham surgery (Sham) or ischemia/reperfusion (I/R; ischemia for 1 hour, reperfusion for $0,1,3,6,12$, or 24 hours) groups were assessed with Western blot. GAPDH served as a loading control. (B) TSLP protein expression of WT and TSLPR ${ }^{-1-}$ mice was assessed in liver (by Western blot) and serum (by ELISA, right) after liver I/R injury (I: 1 hour; R: 6 hours). All data are shown as the mean \pm SEM. $n=5$ in sham groups, $n=6$ in liver I/R groups. NS, no significance. (C and D) TSLP and TSLPR protein expression in primary WT hepatocytes (C) and nonparenchymal cells (D) subjected to hypoxia for 10 hours (1\% oxygen) and then reoxygenation for different time points $(0,2,4,6,8,10$, and 12 hours) (H/R). (E) Primary WT hepatocytes (HC) and nonparenchymal cells (NPC) were cultured either in normal oxygen (control group) or in hypoxia for 10 hours (1\% oxygen) and then reoxygenation for 8 hours (H/R group). TSLP protein levels in supernatant were assessed with Western blot. For Western blot results, figures are representative of data from multiple mice per experimental group or 3 independent in vitro experiments. ELISA data were assessed by unpaired, 2-tailed Student's $t$ test (B).

death during ischemia $(12,13)$ and we and others have demonstrated that autophagy protects from liver I/R injury (12-14). To determine if TSLP regulates protective autophagy during I/R we assessed autophagy markers after liver I/R injury. LC3 and Beclin-1 protein levels increased, while P62 levels decreased during I/R in WT mice. These changes were not observed in $\mathrm{TSLPR}^{-/-}$mice (Figure 3A). During autophagy, autophagosomes can form from new autophagosome formation and/or blockade of autophagosome degradation. To assess these possibilities, we cultured WT and TSLPR ${ }^{-/}$hepatocytes with or without bafilomycin A1, which inhibits autophagolysosomal fusion and degradation, and then subjected the cultured hepatocytes to normoxia or hypoxia/reoxygenation (H/R). LC3 levels increased in WT hepatocytes after both normoxia and H/R conditions when compared with $\mathrm{TSLPR}^{-/-}$hepatocytes after bafilomycin A1 treatment (Figure 3B). We also transfected GFP-LC3 into hepatocytes and quantified the accumulation of GFP-LC3 puncta by confocal microscopy. Consistent with the previous results, the number of GFP-LC3 puncta in WT hepatocytes was greater than that observed in $\mathrm{TSLPR}^{-/-}$hepatocytes under either normoxia or H/R conditions after bafilomycin A1 treatment (Figure 3C). Taken together, these data indicate that TSLP can induce and regulate autophagosome formation in hepatocytes.

Hepatoprotective effects of TSLP in liver $I / R$ injury are not mediated by regulating cytokine levels. The most important functions of TSLP are to induce Th2 responses, regulate Th1/Th2 cell ratios, and promote the differentiation of Th17 cells (5). A number of cytokines are involved in liver I/R injury (3, 15). To ascertain whether the hepatoprotective effects of TSLP in liver I/R injury are mediated by regulating inflammatory cytokine production, we assessed IL- 6 and TNF- $\alpha$ expression in the ischemic hepatic lobe and plasma at 1,3 , and 6 hours of reperfusion. The relative mRNA levels of IL- 6 and TNF- $\alpha$ were markedly elevated at 3 hours; however, no significant difference was observed between the 2 genotypes (Figure 4 , A and C). In plasma, the IL-6 levels were highest at 6 hours after reperfusion, but again there were no significant differences between the 2 genotypes (Figure 4B). Plasma TNF- $\alpha$ levels were not detectable at any reperfusion time point (data not shown). In addition, there were no significant differences between the 2 genotypes for other cytokines representative of Th1, Th2, and Th17 immune responses after 6 hours of reperfusion (Supplemental Figure 1; supplemental material available online with this article; https://doi.org/10.1172/ jci.insight.129013DS1). Thus, the differences in the degree of liver injury between WT and TSLPR ${ }^{-/-}$mice were not associated with higher inflammatory cytokine levels in the absence of TSLP signaling.

TSLP activates the PI3K/Akt pathway in liver I/R injury. Having determined that TSLP protects against liver I/R injury, we next explored the mechanisms underlying the modulation of cellular functions by TSLP. 
A

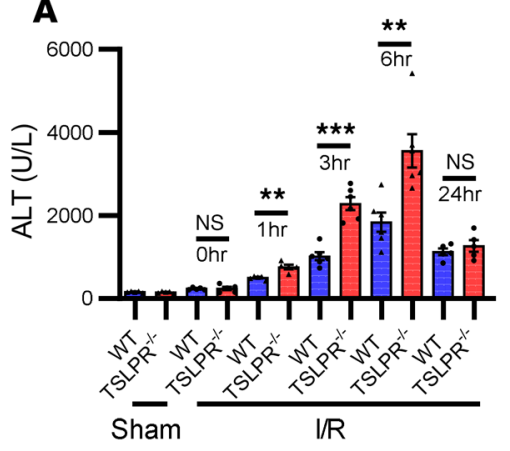

B
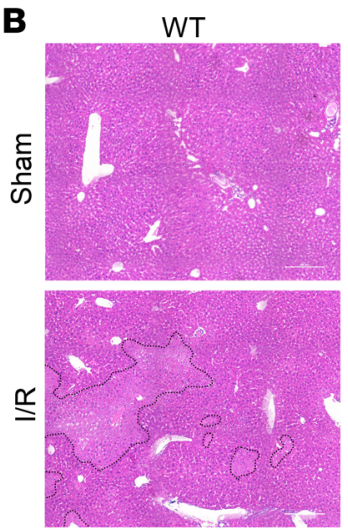

TSLPR $^{-1-}$

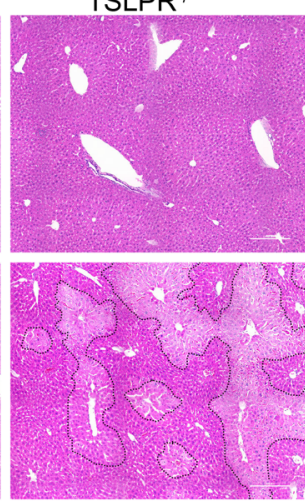

I/R: $1 / 6 \mathrm{hr}$

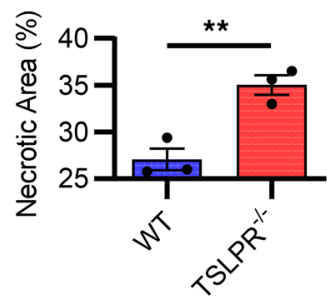

C

F

I/R
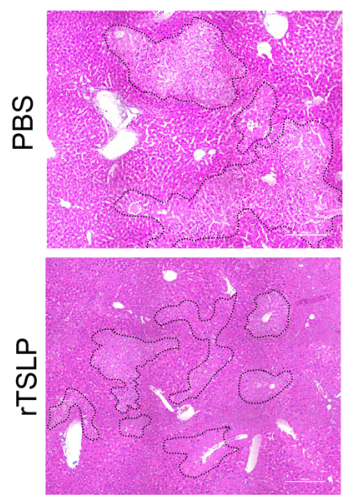

I/R: 1/6hr

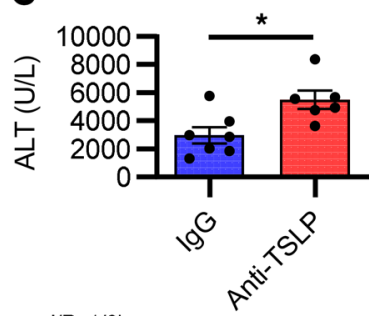

I/R: 1/6hr

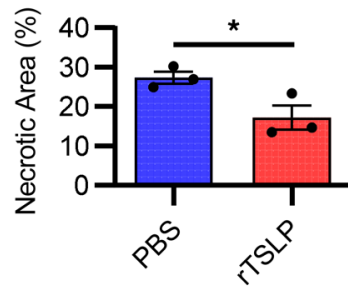

G

I/R: $1 / 6 \mathrm{hr}$
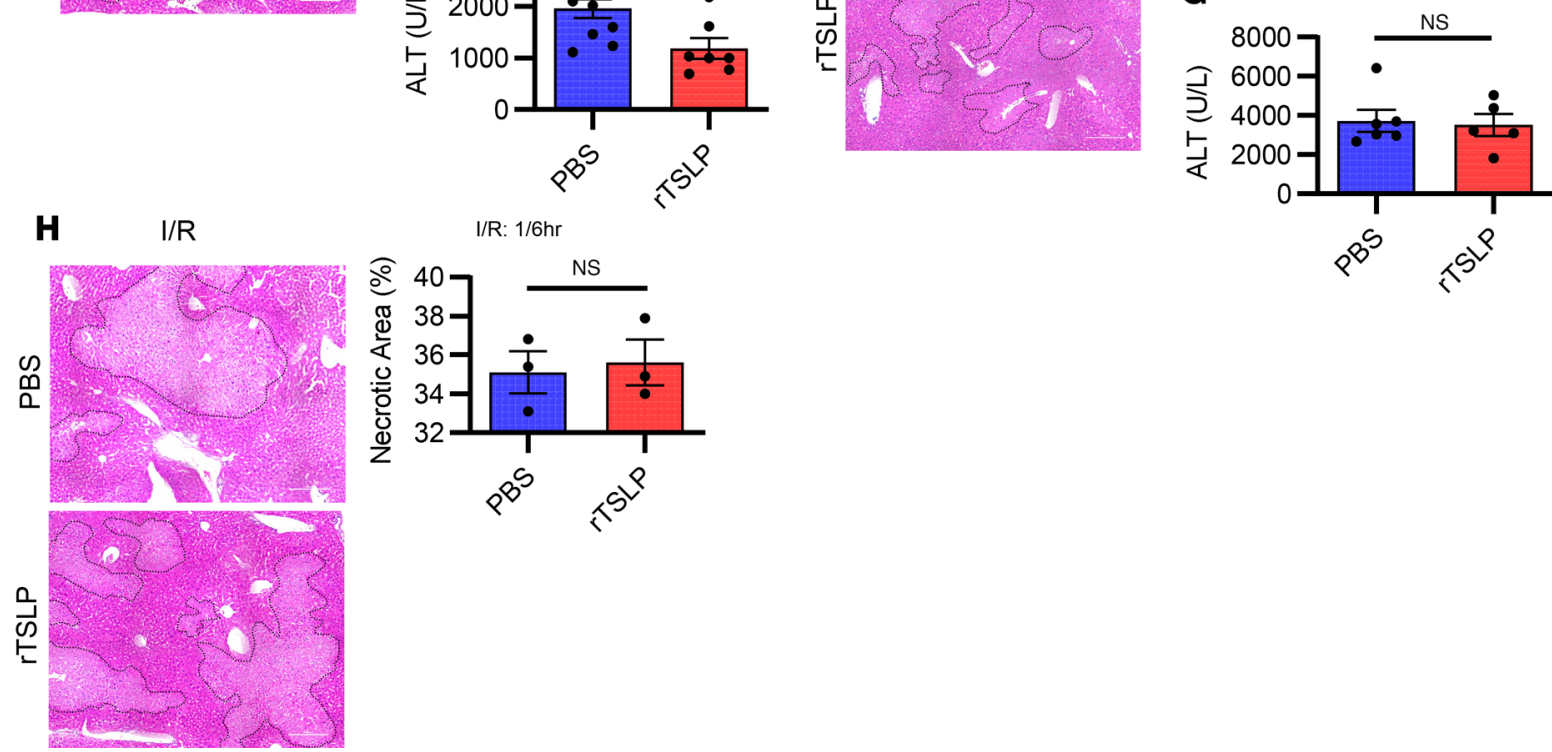

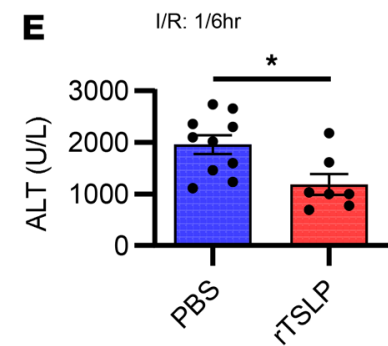

I/R: $1 / 6 \mathrm{hr}$

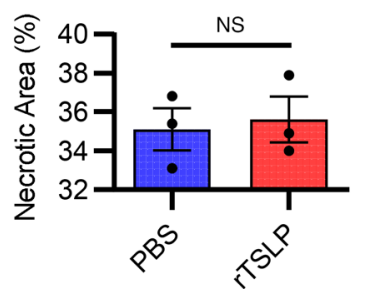

Figure 2. TSLP signaling protects against liver I/R injury. (A) Serum ALT levels of WT and TSLPR ${ }^{-1-}$ mice after sham surgery or liver I/R injury (I: 1 hour; $\mathrm{R}: 0,1,3,6$, or 24 hours). ${ }^{* *} P<0.01,{ }^{* * *} P<0.001 . n=5$ in sham groups, $n=5$ in liver I/R groups (I: 1 hour; R: 0,1, or 24 hours), $n=6$ in liver I/R groups (I: 1 hour; R: 3 or 6 hours). (B) Representative H\&E staining images $(\times 20)$ and necrotic areas of ischemic liver lobes of WT and TSLPR ${ }^{-1-}$ mice at 6 hours after reperfusion or sham controls. Dotted lines indicate measured areas of necrosis, quantified in the bar graph. ${ }^{* *} P<0.01 . n=5$ in sham groups, $n=6$ in liver I/R groups. (C) Serum ALT levels of WT mice after liver I/R injury with IgG or anti-TSLP antibody treatment $(100 \mu \mathrm{g} /$ mouse, i.p. immediately after reperfusion). ${ }^{*} P<0.05$. (D) Representative H\&E staining images $(\times 20)$ and necrotic areas of ischemic liver lobes of WT mice at 6 hours after reperfusion with IgC or anti-TSLP antibody treatment. Dotted lines indicate measured areas of necrosis, quantified in the bar graph. ${ }^{*} P<0.05$. In $\mathbf{C}$ and $\mathbf{D}, n=7$ in $\lg C$ group, $n=$ 6 in anti-TSLP group. (E) Serum ALT levels of WT mice after liver I/R injury with PBS or mouse recombinant TSLP protein (rTSLP) treatment $(2 \mu \mathrm{g} / \mathrm{mouse}$, i.p. immediately after reperfusion). ${ }^{*} P<0.05$. (F) Representative H\&E staining images $(\times 20)$ and necrotic areas of ischemic liver lobes of WT mice at 6 hours after reperfusion with PBS or rTSLP protein treatment. Dotted lines indicate measured areas of necrosis, quantified in the bar graph. ${ }^{*} P<0.05$. In $\mathbf{E}$ and $\mathbf{F}, n=10$ in PBS group, $n=7$ in rTSLP group. (G) Serum ALT levels of TSLPR ${ }^{-1-}$ mice after liver I/R injury with PBS or rTSLP treatment (2 $\mu$ g/mouse, i.p. immediately after reperfusion). (H) Representative H\&E staining images $(\times 20)$ and necrotic areas of ischemic liver lobes of TSLPR $R^{-1-}$ mice at 6 hours after reperfusion with PBS or rTSLP protein treatment. Dotted lines indicate measured areas of necrosis, quantified in the bar graph. In $\mathbf{G}$ and $\mathbf{H}, n=6$ in PBS group, $n=5$ in rTSLP group. All data are shown as the mean \pm SEM. $P$ values by unpaired, 2 -tailed Student's $t$ test (A-H). NS, no significance. 
A

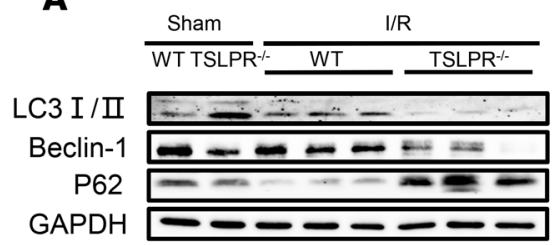

B

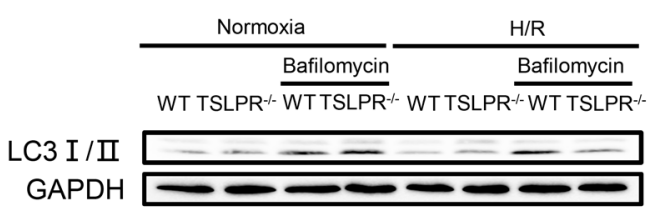

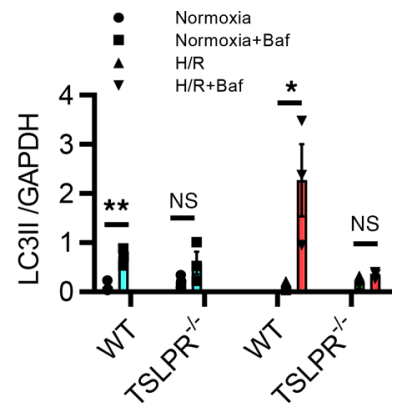

$\mathrm{H} / \mathrm{R}$

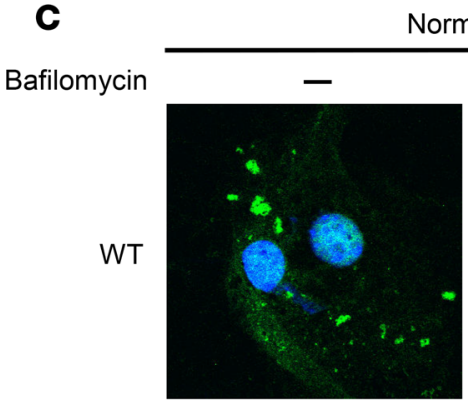

Normoxia
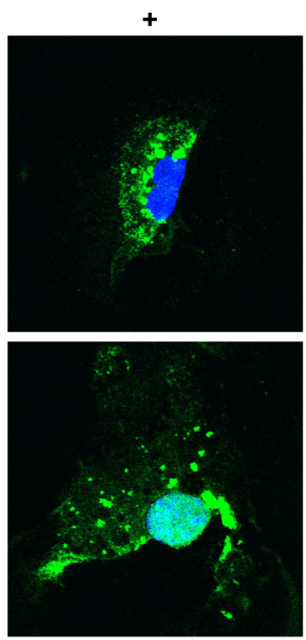
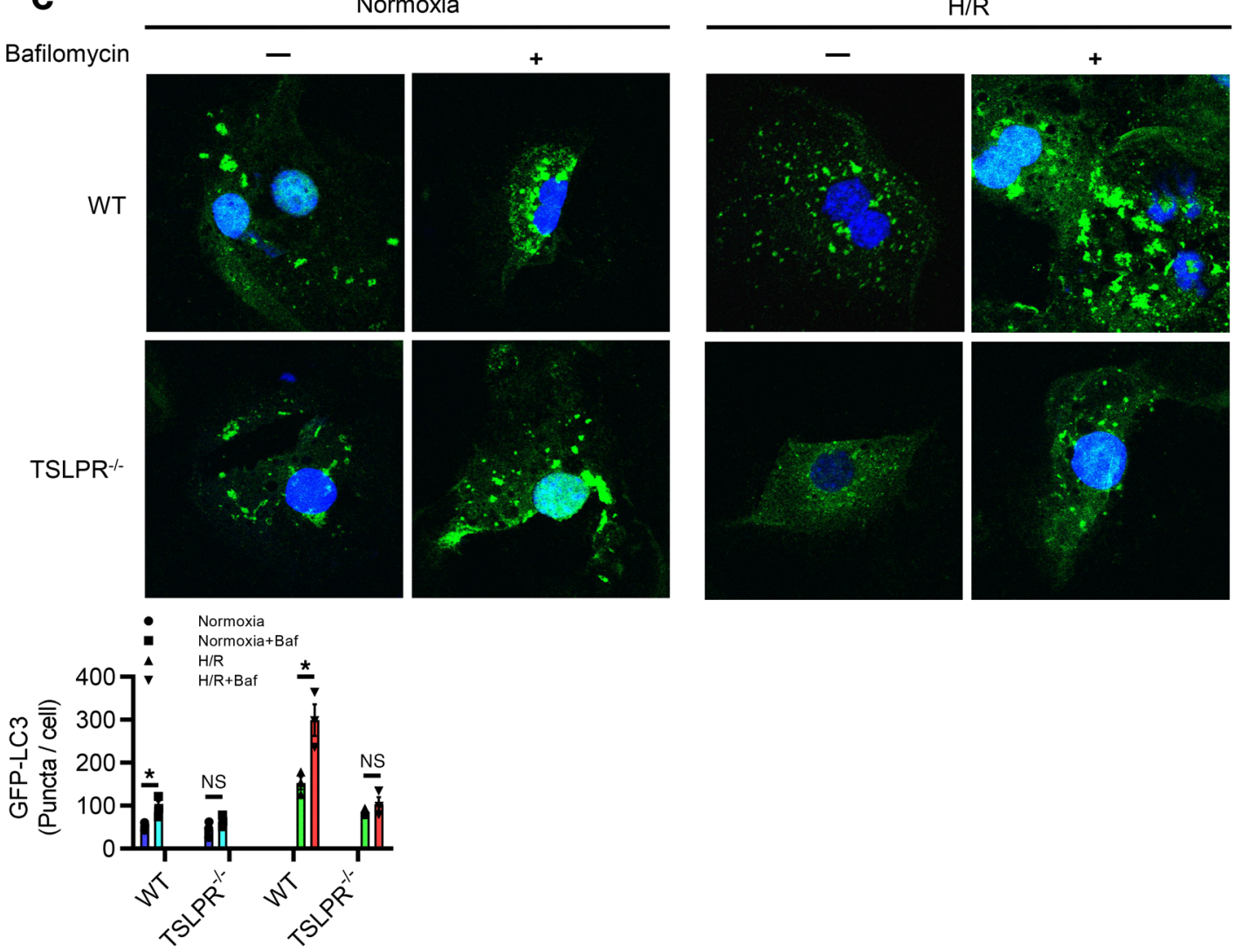

Figure 3. TSLP induces autophagy in vivo and in vitro. (A) Western blot showing the levels of LC3, Beclin-1, and P62 in liver of WT and TSLPR ${ }^{-/-}$mice after liver I/R injury (I: 1 hour; R: 6 hours). $n=5$ in sham groups, $n=6$ in liver I/R groups. (B) Western blot showing the levels of LC3 in whole-cell lysates from WT and TSLPR ${ }^{-/-}$hepatocytes subjected to normoxia or H/R (H: 10 hours; R: 8 hours) with/without bafilomycin A1 (50 nM). Volume of LC3 II bands measured by ImageJ software and represented in bar graph relative to GAPDH loading control. Images are representative of data from at least 3 independent in vitro experiments. ${ }^{* *} P<0.01,{ }^{*} P<0.05$. (C) Confocal microscopy images of WT and TSLPR ${ }^{-/-}$hepatocytes overexpressing GFP-LC3 (green) and Hoechst nuclear stain (blue) and subjected to normoxia or H/R (H: 10 hours; R: 8 hours) and with/without 60-minute bafilomycin A1 (50 nM) treatment (original magnification, $\times 40)$. GFP-LC3 puncta were counted per cell and are represented in the bar graph. ${ }^{*} P<0.05$. All data are shown as the mean \pm SEM. $P$ values by unpaired, 2-tailed Student's $t$ test (B and $\mathbf{C}$ ). For Western blot results, figures are representative of data from multiple mice per experimental group or 3 independent in vitro experiments. NS, no significance.

Several studies have indicated that TSLP can induce Akt phosphorylation and influence apoptosis, proliferation, growth, and survival (16-19). Akt activation has been shown to be a potential therapeutic target in liver I/R injury (20). Consistent with previous studies (20), we found that WT mice exhibited higher liver phosphorylated Akt levels starting at 1 hour after reperfusion that persisted to 6 hours. By 24 hours phosphorylated Akt levels had dropped to similar levels in both WT and TSLPR ${ }^{-1-}$ mice (Figure $5 \mathrm{~A})$. The changes in liver phosphorylated Akt levels over time were consistent with the differences in serum ALT levels between groups described above. In order to ascertain the relationship between TSLP and Akt in liver I/R injury, we assessed Akt in the liver before and after I/R. Phosphorylated Akt levels were lower in WT mice treated with anti-TSLP antibody when compared with IgG after I/R (Figure 5B). However, phosphorylated Akt levels increased in WT mice with rTSLP treatment when compared 

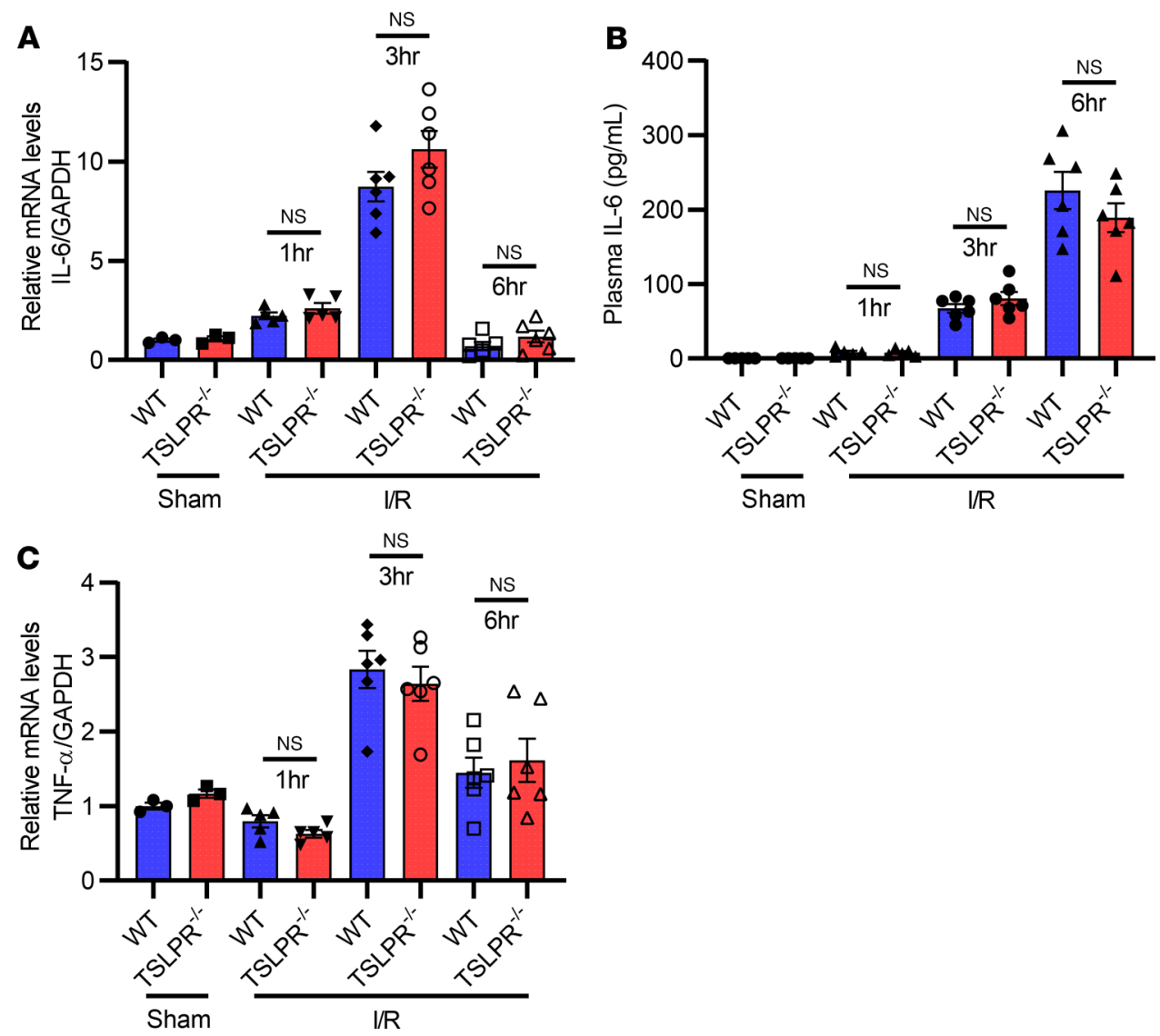

Figure 4. Hepatoprotective effects of TSLP in liver I/R injury are not mediated by regulating cytokine levels. (A) Relative mRNA levels of IL-6 were assessed by qRT-PCR in liver tissues from WT mice and TSLPR ${ }^{-1-}$ mice in sham or I/R (I: 1 hour; R: 1, 3, or 6 hours) groups. (B) Plasma IL-6 levels of WT and TSLPR ${ }^{-/-}$mice after sham surgery or liver I/R injury (I: 1 hour; R: 1 , 3, or 6 hours). $n=5$ in sham groups, $n=5$ in liver I/R groups (I: 1 hour; R: 1 hour), $n=6$ in liver I/R groups (I: 1 hour; R: 1, 3, or 6 hours). (C) Relative mRNA levels of TNF- $\alpha$ were assessed by qRT-PCR in liver tissues from WT mice and TSLPR ${ }^{-1-}$ mice in sham or I/R (I: 1 hour; R: 1, 3, or 6 hours) groups. In $\mathbf{A}$ and $\mathbf{C}$, GAPDH served as a control. Results are expressed as the relative fold increase from 3 experiments compared with control. All data are shown as the mean \pm SEM. $P$ values by unpaired, 2-tailed Student's $t$ test (A-C). NS, no significance.

with PBS (Figure 5C). In TSLPR ${ }^{-/-}$mice, phosphorylated Akt remained at low levels even with rTSLP administration (Figure 5C). Taken together, these data indicate that TSLP can transiently activate the PI3K/Akt pathway during liver I/R.

TSLP induces Akt phosphorylation in vitro. Hepatocytes and nonparenchymal cells cultured alone or together were subjected to H/R (hypoxia, 10 hours; reoxygenation, 8 hours). Phosphorylated Akt levels increased in WT cells when compared with $\mathrm{TSLPR}^{-/-}$following H/R (Figure 6A). In order to determine whether TSLP can directly activate Akt, cultured hepatocytes and nonparenchymal cells were exposed to rTSLP. Consistent with the results in vivo, TSLP induced Akt activation directly in vitro at TSLP doses as low as $10 \mathrm{ng} / \mathrm{mL}$ (Figure 6B). rTSLP-induced ( $100 \mathrm{ng} / \mathrm{mL}$ ) Akt activation peaked at 2 hours (Figure 6C). To assess the roles of TSLPR in nonparenchymal cells and hepatocytes, we measured the levels of total and phosphorylated Akt in primary WT or TSLPR ${ }^{-/-}$hepatocytes and hepatocytes cocultured with nonparenchymal cells that were treated with rTSLP $(100 \mathrm{ng} / \mathrm{mL})$ for 2 hours. Akt activation increased in WT hepatocytes with or without nonparenchymal cells. This was not observed when TSLPR ${ }^{-1-}$ hepatocytes were used in the cultures (Figure 6D). These data indicate that TSLP can induce Akt via the TSLPR in hepatocytes independently of nonparenchymal cells.

TSLP protects against liver I/R injury via the PI3K/ Akt pathway. In order to elucidate whether Akt pathway activation is necessary for TSLP to protect against liver I/R injury, an Akt agonist, insulin-like growth factor-1 (IGF-1) or Akt inhibitor, LY294002, was administered during liver I/R. As expected, IGF-1 induced Akt activation and protected against liver damage after liver I/R $(21,22)$. To determine if Akt activation 
A

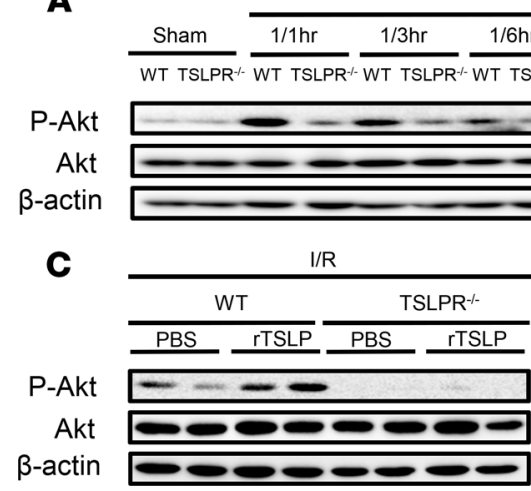

B

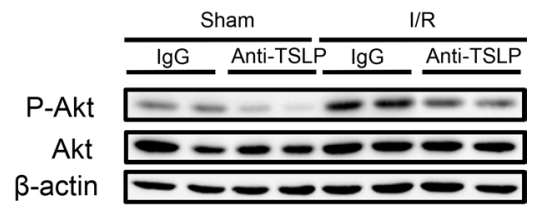

Figure 5. TSLP activates the PI3K/Akt pathway in liver I/R injury. (A) Western blot showing the levels of total and phosphorylated Akt in liver of WT and TSLPR ${ }^{-/-}$mice after liver I/R injury. (B and C) Western blots showing the levels of total and phosphorylated Akt in liver of WT and TSLPR ${ }^{-/-}$mice after liver I/R injury with different treatments: IgC (100 $\mu \mathrm{g} /$ mouse), anti-TSLP (100 $\mu \mathrm{g} /$ mouse), PBS, and $\mathrm{rTSLP}(2 \mu \mathrm{g} /$ mouse) were administered i.p. immediately after reperfusion. Each lane represents a separate animal. The blots shown are representative of 3 experiments with similar results.

was protective in the absence of TSLPR, TSLPR ${ }^{-/}$mice received 2 injections of IGF-1 (100 $\left.\mu \mathrm{g} / \mathrm{kg} / \mathrm{dose}\right)$ subcutaneously: one immediately before the surgical procedure and another immediately after reperfusion. The activation of Akt in the liver was confirmed at 6 hours (Figure 7A). IGF-1 ameliorated liver injury in $\mathrm{TSLPR}^{-/}$mice after liver I/R, as assessed by serum ALT levels and necrosis by histology (Figure 7, B and C). Having determined that rTSLP treatment could protect against liver I/R injury in WT mice, we tested whether inhibiting Akt activation would prevent the protective effects of rTSLP treatment. Akt inhibition with Ly294002 has been shown to exacerbate liver damage during liver I/R injury (23). WT mice were given LY294002 $(0.5 \mathrm{mg} / \mathrm{kg}$ ) plus PBS or LY294002 in addition to rTSLP treatment ( $2 \mu \mathrm{g} / \mathrm{mouse})$ i.p. 30 minutes before surgery and immediately after reperfusion. Phosphorylated Akt levels decreased remarkably with LY294002 with either PBS or rTSLP, indicating that Akt activation was inhibited (Figure 7D). Liver damage was similar between the LY294002 plus PBS and LY294002 plus rTSLP groups, as assessed by serum ALT levels and necrosis by histology (Figure 7, E and F). These results demonstrate that the protective effect of TSLP is lost when Akt activation is inhibited. Furthermore, LC3 I/II protein levels increased obviously in the livers of WT mice undergoing I/R after rTSLP treatment. However, these changes were not observed when Akt activation was inhibited by LY294002 (Figure 7G). Taken together, these findings support the conclusion that TSLP protects against liver I/R injury via the activation of the PI3K/Akt pathway.

\section{Discussion}

TSLP is a cytokine mainly released by epithelial cells that plays important roles in inflammation, autoimmune disease, and cancer (5, 7-10). However, its role in I/R injury was largely unknown. Here, we provide strong evidence that TSLP and its receptor are rapidly upregulated by ischemia in the liver. Acting through its receptor, TSLP protects hepatocytes from necrotic death by activating Akt and promoting autophagy. Thus, we identify TSLP as a potent survival factor for hepatocytes. These findings raise the possibility that TSLP could be used to prevent hepatocellular death induced by ischemic insults.

Previous studies have revealed that TSLP is a key molecule for initiating allergic inflammation such as atopic dermatitis and asthma through DCs and mast cells by triggering inflammatory Th2 responses characterized by high TNF- $\alpha$ and little IL-10 production (5). Recent studies confirmed that TSLP promotes survival of malignant cells and angiogenesis in ischemic stroke $(19,24)$. Our study is the first to our knowledge to document the role of TSLP in sterile inflammation induced by I/R. More specifically, our study links TSLP to the activation of the PI3K/Akt pathway through its canonical receptor in the protection of hepatocytes from ischemic injury.

TSLP protein expression is abundant in liver, and especially in hepatocytes. In a previous study, TSLP secreted by hepatocytes and keratinocytes of HCV-infected patients with cryoglobulinemic vasculitis contributed to the pathogenesis of vasculitis (25). Mechanical injury, the proinflammatory milieu, and proteases, such as trypsin and papain, were found to cause TSLP production and release from the epithelial 
A

$$
\begin{array}{r}
\text { A-Akt } \\
\text { Akt } \\
\text { GAPDH }
\end{array}
$$$$
\text { C }
$$

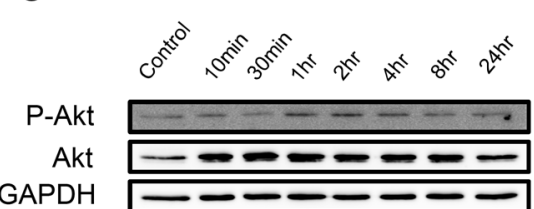

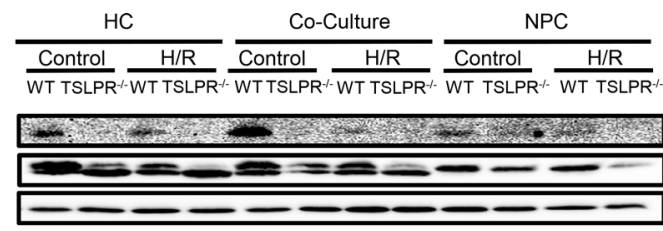

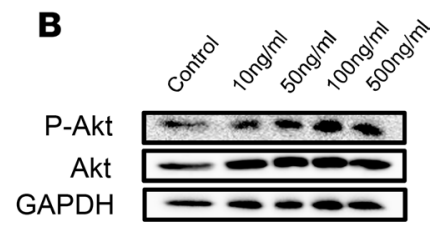

Figure 6. TSLP induces Akt phosphorylation in vitro. (A) Western blot showing the levels of total and phosphorylated Akt in primary WT and TSLPR ${ }^{-1-}$ hepatocytes $(\mathrm{HC}$ ) or nonparenchymal cells (NPC) subjected to control or hypoxia for 10 hours ( $1 \%$ oxygen) and then reoxygenation for 8 hours (H/R). For coculture group, HC and NPC were cultured together and then subjected to control or H/R. (B) Western blot showing the levels of total and phosphorylated Akt in primary WT HC after receiving different doses of $\mathrm{rTSLP}(10 \mathrm{ng} / \mathrm{mL}, 50 \mathrm{ng} / \mathrm{mL}, 100 \mathrm{ng} / \mathrm{mL}$, and $500 \mathrm{ng} / \mathrm{mL}$ ) treatment for 2 hours. (C) Western blot showing the levels of total and phosphorylated Akt in primary WT HC after receiving rTSLP $(100 \mathrm{ng} / \mathrm{mL})$ treatment at different time points (10 minutes, 30 minutes, 1 hour, 2 hours, 4 hours, 8 hours, and 24 hours). (D) Western blot showing the levels of total and phosphorylated Akt in primary WT or TSLPR ${ }^{-1-} \mathrm{HC}$ and HC cocultured with NPC after receiving rTSLP $(100 \mathrm{ng} / \mathrm{mL})$ treatment for 2 hours. The blots shown are representative of 3 experiments with similar results.

compartments $(5,26)$. During liver I/R injury, there are 2 phases: the ischemic phase, which is characterized by oxidative stress and hepatocyte ischemic stress and adaptation; and the subsequent reperfusion phase, which is characterized by cell death and inflammation. In the present study, we observed that TSLP and TSLPR protein expression increased markedly during the ischemic phase of liver I/R injury. While little TSLP is released systemically, it is clear based on the use of a TSLP-neutralizing antibody that TSLP can act in an autocrine or paracrine manner to trigger signaling in ischemic hepatocytes. It is possible that most of the TSLP released by hepatocytes is taken up through interaction with TSLPR locally. How ischemia leads to an upregulation of hepatocyte TSLP and TSLPR levels is unknown.

TSLP is known to exacerbate tissue injury in many infectious diseases by activating DC-primed naive $\mathrm{T}$ cells to produce proallergic cytokines (IL-4, IL-5, IL-13, TNF- $\alpha$ ) while downregulating IL-10 and IFN- $\gamma$ (27). In a halothane-induced liver injury (HILI) mouse model, TSLP and IL-4 mediate the pathogenesis of liver injury. The severity of HILI was reduced in mice deficient in either TSLPR or IL-4 and was accompanied by decreases in serum levels of eotaxins and hepatic eosinophilia (28). Another study revealed that hepatocyte-derived TSLP conditions DCs to drive Th17 differentiation. Furthermore, blockade of TSLP released by HCV-infected hepatocytes may suppress the induction/maintenance of hepatic Th17 responses and halt the progression of chronic liver disease to fibrosis and liver failure (29). The protective effects of TSLP in our model may relate to the acute nature of the I/R insult and the presence or absence of other inflammatory mediators in the microenvironment. In addition, our data point to the impact of TSLP directly acting on hepatocytes as opposed to the activation of cytokine release by other cells.

The best-characterized function of TSLP is to induce Th2 responses defined by high amounts of IL-4, IL-5, IL-13, and TNF- $\alpha$, but not IL-10 or IFN- $\gamma(5,27)$. In allergic inflammation, Th2 responses initiate and exacerbate tissue injury (30). However, in liver I/R injury, previous studies have shown that Th2 responses can protect against liver damage $(31,32)$. To determine whether the hepatoprotective effect of TSLP in liver I/R injury is related to Th2 cytokines, we assessed inflammatory cytokine expression in the ischemic liver. According to our data, there were no significant differences between WT mice and TSLPR ${ }^{-/}$mice in Th1, Th2, and Th17 responses, supporting the idea that TSLP effects in I/R are not through the upregulation of type 2 cytokines.

The PI3K/Akt signaling pathway regulates diverse cellular functions including metabolism, growth, proliferation, survival, transcription, and protein synthesis (33). Many studies have demonstrated that the PI3K/Akt signaling pathway plays an important role in liver I/R injury by inhibiting proapoptotic signals, inflammation, and mitochondria-produced ROS, while at the same time promoting antiapoptotic effects, cell growth, and vasoregulation. Akt is thought to be a potential therapeutic target for liver I/R injury $(20,34)$. Our previous study revealed that carbon monoxide activates NF- $\mathrm{kB}$ via ROS generation and Akt pathways to protect against death of hepatocytes (35). Another study confirmed that ischemic preconditioning of the murine liver protects 
A
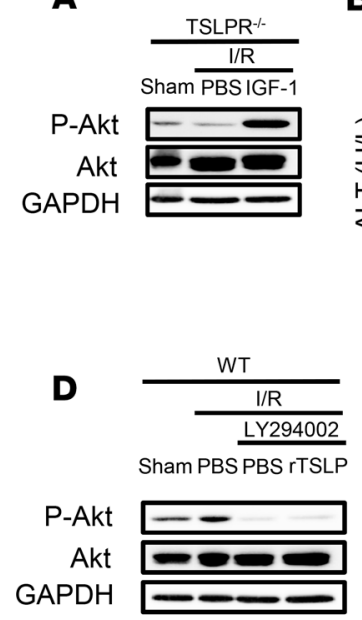

B
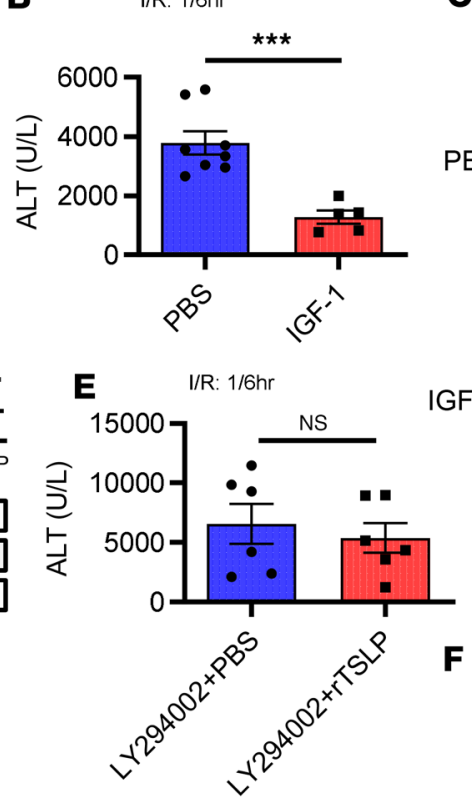

G

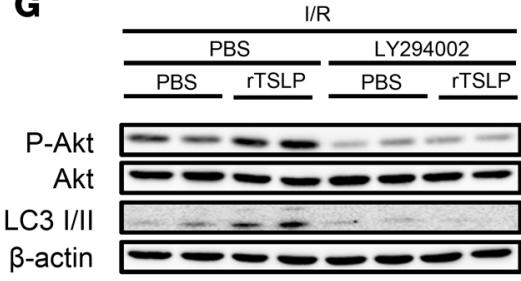

C

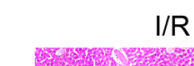

$\mathrm{I} / \mathrm{R}$

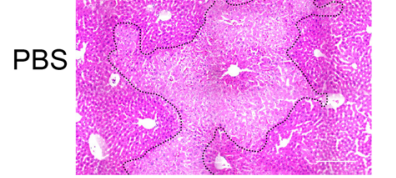

GF-1

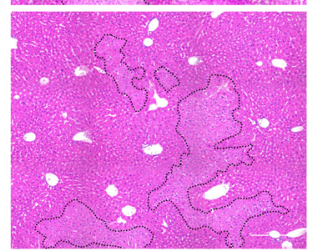

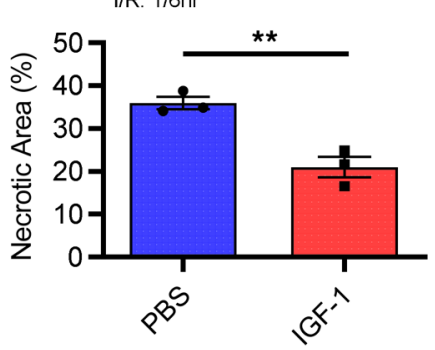

I/R

LY294002 +PBS
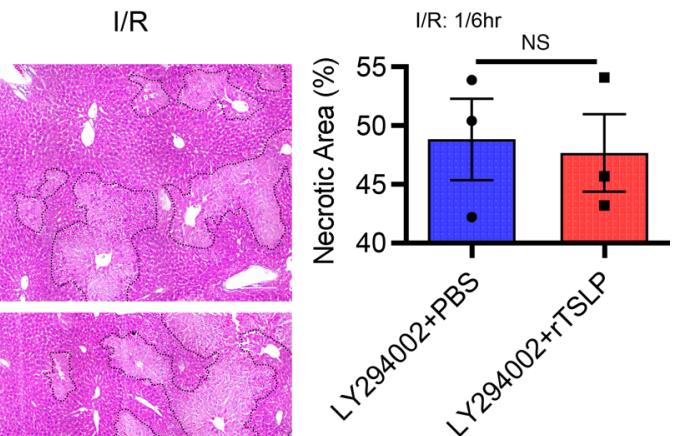

LY294002+rTSLP

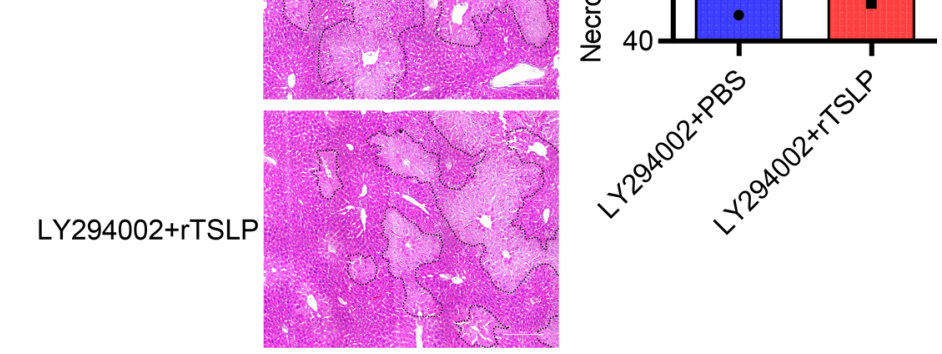

I/R: $1 / 6 h r$

Figure 7. TSLP protects against liver I/R injury via PI3K/Akt pathway. (A) Western blot showing the levels of total and phosphorylated Akt in liver of TSLPR $^{-1-}$ mice after liver I/R injury (I: 1 hour; R: 6 hours) with PBS or Akt agonist insulin-like growth factor-1 (IGF-1) (2 doses, $100 \mu \mathrm{g} / \mathrm{kg} / \mathrm{dose}$, subcutaneous injection, one dose starting immediately before surgical procedure, another dose starting immediately after reperfusion) treatment. (B) Serum ALT levels of TSLPR ${ }^{-1-}$ mice after liver I/R injury with PBS or IGF-1 treatment. ${ }^{* *} P<0.001$. (C) Representative H\&E staining images ( $\left.\times 20\right)$ and necrotic areas of ischemic liver lobes of TSLPR ${ }^{-/-}$mice at 6 hours after reperfusion with PBS or IGF-1 treatment. Dotted lines indicate measured areas of necrosis, quantified in the bar graph. ${ }^{* *} P<0.01$. In A-C, $n=8$ in PBS group, $n=5$ in IGF-1 group. (D) Western blot showing the levels of total and phosphorylated Akt in liver of WT mice after liver I/R injury with PBS or LY294002 plus PBS or LY294002 plus rTSLP treatment (LY294002 [0.5 mg/kg] was administered i.p. 30 minutes before surgery; PBS and rTSLP [2 $\mu \mathrm{g} /$ mouse] were administered i.p. immediately after reperfusion). (E) Serum ALT levels of WT mice after liver I/R injury with LY294002 plus PBS or LY294002 plus rTSLP treatment. (F) Representative H\&E staining images ( $\times 20)$ and necrotic areas of ischemic liver lobes of WT mice at 6 hours after reperfusion with LY294002 plus PBS or LY294002 plus rTSLP treatment. Dotted lines indicate measured areas of necrosis, quantified in on the bar graph. In D-F, $n=6$ per group. (C) Western blot showing the levels of total Akt, phosphorylated Akt, and LC3 in liver of WT mice after liver I/R injury with PBS or LY294002 plus PBS or rTSLP treatment. The Western blots shown are representative of 3 experiments with similar results. All data are shown as the mean \pm SEM. $P$ values by unpaired, 2-tailed Student's $t$ test (B, C, E, and F). NS, no significance.

through the Akt kinase pathway (36). TSLP has been confirmed to stimulate normal and malignant B lymphopoietic proliferation by activating Akt signaling cascades $(16,19)$. Consistent with these previous studies (20), Akt was phosphorylated and activated in WT mice after liver I/R injury. Thus, TSLP appears to support Akt signaling and administration of exogenous TSLP can further activate Akt to provide even greater protection. Taken together, our results confirm that TSLP protects against liver I/R injury via the PI3K/Akt pathway.

As demonstrated, autophagy can maintain cell homeostasis and ensures cell survival under stressful conditions via regulating the degradation of damaged cytoplasm and mitochondria (37). Many studies show that autophagy plays a pivotal role in maintaining mitochondrial function and cell survival after liver I/R injury (38). Our data indicated that TSLP upregulates autophagy during liver I/R to protect against liver damage. This result is different from the function of TSLP in chronic inflammatory disease, where TSLP was observed to reduce autophagy and serve as a fundamental contributor to chronic obstructive pulmonary disease (COPD) (39). The explanation for the difference may relate to the acute nature of $I / R$ injury versus the slower induction of type 2 immune responses needed for many of the more delayed effects of TSLP in chronic disease models. 
In conclusion, the present study reveals that TSLP protects against liver I/R injury via activation of the PI3K/Akt pathway and by inducing autophagy. Because exogenous TSLP prevented I/R-induced liver injury through these pathways even in WT mice, it is possible that TLSP could be used to prevent ischemia-induced liver damage. In addition, these findings broaden our understanding of the functions of TSLP in acute sterile inflammation induced by I/R injury. Furthermore, we also found that liver injury was higher at 6 hours after acetaminophen $\left(400 \mathrm{mg} / \mathrm{kg}\right.$ ) administration in TSLPR ${ }^{-1-}$ mice (Supplemental Figure 2 ), suggesting that the protective effects of TSLP signaling in the liver may extend to other models of injury.

\section{Methods}

Animals. TSLPR ${ }^{-1-}$ (on a C57BL/6J background) mice were a gift from Richard Locksley (UCSF, San Francisco, California, USA) and were bred in our facility. C57BL/6J WT mice were purchased from Jackson Laboratory. All mice used in the study were male and 8-12 weeks old.

Reagents. Western blot antibodies: TSLP (catalog PA5-20321) and TSLPR (catalog PA5-20380) antibodies were from Invitrogen; GAPDH (catalog ab8245) and $\beta$-actin (catalog ab8226) antibodies were from Abcam; Autophagy Antibody Sampler Kit (LC3 antibody, Beclin-1 antibody, P62 antibody; 4445), phospho-Akt antibody (S473) (catalog 9271), and Akt antibody (catalog 9272) were from Cell Signaling Technology. Mouse TSLP Quantikine ELISA Kit (catalog MTLP00) was from R\&D Systems. The reagents for animal treatment and cell culture were as follows: rat IgG (azide free) (catalog 6-001-F), anti-TSLP antibody (catalog MAB555), mouse rTSLP (catalog 555-TS-101), and recombinant mouse IGF-1 (catalog 791-MG-050) were from R\&D Systems. LY294002 (catalog L9908) and bafilomycin A1 (catalog 19-148) were from Sigma-Aldrich.

Mouse liver $I / R$ injury model and animal treatment. A nonlethal model of segmental (70\%) hepatic warm I/R was used as described previously (40). Briefly, all structures in the portal triad (hepatic artery, portal vein, bile duct) to the left and median liver lobes were occluded with a microvascular clamp for 60 minutes and reperfusion was initiated by clamp removal. The temperature of the mice during ischemia was maintained at $33^{\circ} \mathrm{C}$ using a warming incubator chamber. Sham animals underwent anesthesia, laparotomy, and exposure of the portal triad without hepatic ischemia. Animals were sacrificed at 6 hours after reperfusion to obtain serum and liver samples. IgG, anti-TSLP antibody (100 $\mu \mathrm{g} / \mathrm{mouse})$, PBS, and mouse rTSLP (2 $\mu \mathrm{g} /$ mouse) were administered i.p. immediately after reperfusion. IGF-1 was administered subcutaneously $(100 \mu \mathrm{g} / \mathrm{kg} /$ dose $) 1$ minute before the surgical procedure and immediately after reperfusion. LY294002 (0.5 $\mathrm{mg} / \mathrm{kg}$, i.p.) was administered 30 minutes before surgery.

Serum sample assays. Serum TSLP concentrations were assessed by Mouse TSLP Quantikine ELISA Kit following the manufacturer's instructions. ALT levels were determined by the DRI-CHEM 4000 Chemistry Analyzer System (Heska).

Histological analysis. Mice were euthanized after designated treatment. The right upper lung lobe and the left lateral lobe of the liver were perfused with PBS and fixed in $2 \%$ paraformaldehyde (PFA). Tissues were then placed in 2\% PFA for an additional 2 hours and then switched to $30 \%$ sucrose in distilled water for 24 hours. The tissue was then slowly frozen in liquid nitrogen-cooled 2-methylbutane according to a standardized protocol for cryopreservation. Cryostat sections of the tissues $(6 \mu \mathrm{m})$ were stained with $\mathrm{H} \& \mathrm{E}$ to evaluate histopathologic cumulative changes among treatment groups. Images of 5 randomly selected fields were acquired using an Olympus Provis light microscope with $\times 20$ magnification. Necrotic areas were analyzed by ImageJ software (NIH).

Hepatocyte and nonparenchymal cell isolation and culture. Hepatocytes were isolated as described previously (41). Mice were perfused by an in situ collagenase (type VI, Worthington) technique. Hepatocytes were separated from nonparenchymal cells and purity exceeded $99 \%$ by light microscopy. The viability was greater than $95 \%$ as measured by trypan blue exclusion. Hepatocytes were cultured as described previously (41). Nonparenchymal cells were isolated and cultured as described previously (12). Nonparenchymal cells were subjected to differential centrifugation and purified without hepatocytes as determined by light microscopy. Hepatocytes $(150,000$ cells $/ \mathrm{mL})$ and nonparenchymal cells $(750,000$ cells $/ \mathrm{mL})$ were plated on gelatin-coated culture plates or coverslips coated with collagen I (BD Pharmingen) in Williams medium E with $10 \%$ calf serum, $15 \mathrm{mM}$ HEPES, $1 \mu \mathrm{M}$ insulin, $2 \mathrm{mM}$ L-glutamine, penicillin $(100 \mathrm{U} / \mathrm{mL})$, and streptomycin $(100 \mathrm{U} / \mathrm{mL})$. Cells were allowed to attach to plates overnight, and the culture medium was replaced with fresh medium before the cells were treated for experiments.

Comparative PCR analysis. The median lobe of the liver was frozen in liquid nitrogen and stored at $-80^{\circ} \mathrm{C}$ for comparative PCR analysis. Total RNA was extracted with the RNeasy mini extraction kit (Qiagen) 
according to the manufacturer's instructions. From $1 \mu \mathrm{g}$ of RNA and with oligo-dT primers (Qiagen) and Omniscript reverse transcriptase (Qiagen), complementary DNA (cDNA) was generated and used for realtime PCR analysis. SYBR Green PCR master mix (PE Applied Biosystems) was used to prepare the PCR reaction mixes. Two-step, real-time reverse transcription PCR (RT-PCR) was performed with forward and reverse primer pairs prevalidated and specific for IL-4, IL-5, IL-6, IL-12, IL-13, IL-17A, TNF- $\alpha$, and IFN- $\gamma$ (Qiagen). All samples were assayed in duplicate, and normalized to $\beta$-actin or GAPDH mRNA abundance.

Western blot. For in vitro experiments, hepatocytes were washed with cold PBS, collected in lysis buffer (Cell Signaling Technology), sonicated, and centrifuged (16,000 $g$ for 15 minutes), after which the supernatant was collected. For in vivo experiments, snap-frozen liver (ischemia lobe) was homogenized in lysis buffer and centrifuged (16,000 $\mathrm{g}$ for 15 minutes), after which the supernatant was collected. Protein concentrations were determined with the bicinchoninic acid (BCA) protein assay kit (Thermo Fisher Scientific). Loading buffer was added to the samples, which were then resolved by $10 \%$ or $15 \%$ sodium dodecyl sulfate-polyacrylamide gel electrophoresis (SDS-PAGE). Samples were then transferred to a polyvinylidene difluoride membrane at 250 $\mathrm{mA}$ for 2 hours. The membrane was blocked in 5\% milk for 1 hour and then incubated with primary antibody in 1\% milk overnight. Membranes were washed in Tris-buffered saline-Tween 20 (TBS-T) for 10 minutes, incubated with horseradish peroxidase-conjugated (HRP-conjugated) secondary antibody for 1 hour, and then washed for 1 hour in TBS-T, before being developed for chemiluminescence (Thermo Fisher Scientific). The chemiluminescent signals were acquired and quantified with the ChemiDoc MP Imaging System (Bio-Rad).

Autophagic flux. Autophagic flux was performed and measured as described previously (12). Primary hepatocytes were cultured under hypoxic conditions (1\% oxygen for 10 hours) following reoxygenation (normoxic conditions for 8 hours) with bafilomycin A1 (50 nM) treatment for 1 hour during reoxygenation. LC3 I/II conversion was detected in whole-cell lysates by Western blot. Another culture of primary hepatocytes was transfected with GFP-LC3 adenovirus before hypoxia. Cells transfected with GFP-LC3 were imaged with a Zeiss LSM510 laser-scanning confocal microscope. GFP-LC3 puncta were counted for 30 cells/treatment.

Statistics. All data are shown as mean \pm standard error of the mean (SEM). Data analysis was performed using GraphPad Prism software. Comparisons between 2 experimental groups were performed by 2-tailed Student's $t$ test. A statistically significant difference was considered when $P<0.05$.

Study approval. Animal protocols were approved by the Animal Care and Use Committee of the University of Pittsburgh, and the experiments were performed in adherence to the NIH Guide for the Care and Use of Laboratory Animals (National Academies Press, 2011).

\section{Author contributions}

SL, MD, MJS, and TRB designed the study. SL, ZY, MD, and TRB generated data and wrote the manuscript. SL, ZY, CY, WL, ZL, NMS, and PL conducted the experiments. All authors reviewed and edited the manuscript.

\section{Acknowledgments}

This work was supported by NIH grant R35-GM127027 (to TRB), Medical Excellence Award funded by the Creative Research Development Grant from the First Affiliated Hospital of Guangxi Medical University (to SL), and National Natural Science Foundation of China grant no. 81960358 (to SL). We thank Lauryn Kohut, Hong Liao, Danielle Reiser, Wentao Gao, and Richard A. Shapiro for technical assistance with in vivo and in vitro experiments.

Address correspondence to: Timothy R. Billiar, Department of Surgery, University of Pittsburgh, Pittsburgh, Pennsylvania 15213, USA. Phone: 412.647.1749; Email: billiartr@upmc.edu.

1. Nastos C, et al. Global consequences of liver ischemia/reperfusion injury. Oxid Med Cell Longev. 2014;2014:906965.

2. van Riel WG, van Golen RF, Reiniers MJ, Heger M, van Gulik TM. How much ischemia can the liver tolerate during resection? Hepatobiliary Surg Nutr. 2016;5(1):58-71.

3. Peralta C, Jiménez-Castro MB, Gracia-Sancho J. Hepatic ischemia and reperfusion injury: effects on the liver sinusoidal milieu. J Hepatol. 2013;59(5):1094-1106.

4. Leonard WJ. TSLP: finally in the limelight. Nat Immunol. 2002;3(7):605-607.

5. Ziegler SF, Roan F, Bell BD, Stoklasek TA, Kitajima M, Han H. The biology of thymic stromal lymphopoietin (TSLP). Adv Pharmacol. 2013;66:129-155.

6. Pandey A, et al. Cloning of a receptor subunit required for signaling by thymic stromal lymphopoietin. Nat Immunol. 
2000;1(1):59-64.

7. Gauvreau GM, et al. Effects of an anti-TSLP antibody on allergen-induced asthmatic responses. $N$ Engl J Med. 2014;370(22):2102-2110.

8. Piliponsky AM, et al. Thymic stromal lymphopoietin improves survival and reduces inflammation in sepsis. Am J Respir Cell Mol Biol. 2016;55(2):264-274.

9. De Monte L, et al. Intratumor T helper type 2 cell infiltrate correlates with cancer-associated fibroblast thymic stromal lymphopoietin production and reduced survival in pancreatic cancer. J Exp Med. 2011;208(3):469-478.

10. Moret FM, Hack CE, van der Wurff-Jacobs KM, Radstake TR, Lafeber FP, van Roon JA. Thymic stromal lymphopoietin, a novel proinflammatory mediator in rheumatoid arthritis that potently activates $\mathrm{CD} 1 \mathrm{c}^{+}$myeloid dendritic cells to attract and stimulate T cells. Arthritis Rheumatol. 2014;66(5):1176-1184.

11. Headley MB, Zhou B, Shih WX, Aye T, Comeau MR, Ziegler SF. TSLP conditions the lung immune environment for the gener ation of pathogenic innate and antigen-specific adaptive immune responses. J Immunol. 2009;182(3):1641-1647.

12. Lei Z, et al. cGAS-mediated autophagy protects the liver from ischemia-reperfusion injury independently of STING. $A m J$ Physiol Gastrointest Liver Physiol. 2018;314(6):G655-G667.

13. Cardinal J, Pan P, Tsung A. Protective role of cisplatin in ischemic liver injury through induction of autophagy. Autophagy. 2009;5(8):1211-1212.

14. Wang JH, et al. Autophagy suppresses age-dependent ischemia and reperfusion injury in livers of mice. Gastroenterology. 2011;141(6):2188-2199.e6.

15. Abu-Amara M, Yang SY, Tapuria N, Fuller B, Davidson B, Seifalian A. Liver ischemia/reperfusion injury: processes in inflammatory networks--a review. Liver Transpl. 2010;16(9):1016-1032.

16. Francis OL, et al. A novel xenograft model to study the role of TSLP-induced CRLF2 signals in normal and malignant human B lymphopoiesis. Haematologica. 2016;101(4):417-426.

17. Dong J, et al. Inflammatory cytokine TSLP stimulates platelet secretion and potentiates platelet aggregation via a TSLPR-dependent PI3K/Akt signaling pathway. Cell Physiol Biochem. 2015;35(1):160-174.

18. Zhong J, et al. TSLP signaling pathway map: a platform for analysis of TSLP-mediated signaling. Database (Oxford). 2014;2014:bau007.

19. Tasian SK, et al. Aberrant STAT5 and PI3K/mTOR pathway signaling occurs in human CRLF2-rearranged B-precursor acute lymphoblastic leukemia. Blood. 2012;120(4):833-842.

20. Covington SM, Bauler LD, Toledo-Pereyra LH. Akt: a therapeutic target in hepatic ischemia-reperfusion injury. J Invest Surg. 2017;30(1):47-55.

21. Casillas-Ramírez A, et al. Insulin-like growth factor and epidermal growth factor treatment: new approaches to protecting steatotic livers against ischemia-reperfusion injury. Endocrinology. 2009;150(7):3153-3161.

22. Romanelli RJ, LeBeau AP, Fulmer CG, Lazzarino DA, Hochberg A, Wood TL. Insulin-like growth factor type-I receptor internalization and recycling mediate the sustained phosphorylation of Akt. J Biol Chem. 2007;282(31):22513-22524.

23. Carini R, et al. Role of phosphatidylinositol 3-kinase in the development of hepatocyte preconditioning. Gastroenterology. 2004;127(3):914-923.

24. Yu X, et al. TSLP/TSLPR promote angiogenesis following ischemic stroke via activation of the PI3K/AKT pathway. Mol Med Rep. 2018;17(2):3411-3417.

25. Sansonno D, Russi S, Sansonno S, Pavone F, Dammacco F. Thymic stromal lymphopoietin in hepatitis C virus-related cryoglobulinemic vasculitis: gene expression level and protein distribution. Arthritis Res Ther. 2015;17:62.

26. Takai T. TSLP expression: cellular sources, triggers, and regulatory mechanisms. Allergol Int. 2012;61(1):3-17.

27. Ziegler SF, Artis D. Sensing the outside world: TSLP regulates barrier immunity. Nat Immunol. 2010;11(4):289-293.

28. Proctor WR, et al. Thymic stromal lymphopoietin and interleukin-4 mediate the pathogenesis of halothane-induced liver injury in mice. Hepatology. 2014;60(5):1741-1752.

29. Lee HC, et al. Hepatitis C virus promotes T-helper (Th)17 responses through thymic stromal lymphopoietin production by infected hepatocytes. Hepatology. 2013;57(4):1314-1324.

30. Verstraete K, et al. Structure and antagonism of the receptor complex mediated by human TSLP in allergy and asthma. Nat Commun. 2017;8:14937.

31. Hamada T, et al. Cyclooxygenase-2 deficiency enhances Th2 immune responses and impairs neutrophil recruitment in hepatic ischemia/reperfusion injury. J Immunol. 2008;180(3):1843-1853.

32. Pulitanò C, et al. Reduced severity of liver ischemia/reperfusion injury following hepatic resection in humans is associated with enhanced intrahepatic expression of Th2 cytokines. Hepatol Res. 2006;36(1):20-26.

33. Manning BD, Toker A. AKT/PKB signaling: navigating the network. Cell. 2017;169(3):381-405.

34. Zhang R, Zhang L, Manaenko A, Ye Z, Liu W, Sun X. Helium preconditioning protects mouse liver against ischemia and reperfusion injury through the PI3K/Akt pathway. J Hepatol. 2014;61(5):1048-1055.

35. Kim HS, Loughran PA, Rao J, Billiar TR, Zuckerbraun BS. Carbon monoxide activates NF-kappaB via ROS generation and Akt pathways to protect against cell death of hepatocytes. Am J Physiol Gastrointest Liver Physiol. 2008;295(1):G146-G152.

36. Izuishi K, et al. Ischemic preconditioning of the murine liver protects through the Akt kinase pathway. Hepatology. 2006;44(3):573-580.

37. Wang K. Autophagy and apoptosis in liver injury. Cell Cycle. 2015;14(11):1631-1642.

38. Go KL, Lee S, Zendejas I, Behrns KE, Kim JS. Mitochondrial dysfunction and autophagy in hepatic ischemia/reperfusion injury. Biomed Res Int. 2015;2015:183469.

39. Roscioli E, et al. The uncoupling of autophagy and zinc homeostasis in airway epithelial cells as a fundamental contributor to COPD. Am J Physiol Lung Cell Mol Physiol. 2017;313(3):L453-L465.

40. Tsung A, et al. The nuclear factor HMGB1 mediates hepatic injury after murine liver ischemia-reperfusion. $J$ Exp Med. 2005;201(7):1135-1143.

41. Sun Q, et al. Redox-dependent regulation of hepatocyte absent in melanoma 2 inflammasome activation in sterile liver injury in mice. Hepatology. 2017;65(1):253-268. 